TRANSACTIONS OF THE

AMERICAN MATHEMATICAL SOCIETY

Volume 357 , Number 7 , Pages 2723-2738

S 0002-9947(04)03560-3

Article electronically published on July 16, 2004

\title{
BRAUER GROUPS OF GENUS ZERO EXTENSIONS OF NUMBER FIELDS
}

\author{
JACK SONN AND JOHN SWALLOW
}

\begin{abstract}
We determine the isomorphism class of the Brauer groups of certain nonrational genus zero extensions of number fields. In particular, for all genus zero extensions $E$ of the rational numbers $\mathbb{Q}$ that are split by $\mathbb{Q}(\sqrt{2})$, $\operatorname{Br}(E) \cong \operatorname{Br}(\mathbb{Q}(t))$.
\end{abstract}

\section{INTRODUCTION}

Let $K$ be a countable field, and let $\operatorname{Br}(K)$ be its Brauer group. Then $\operatorname{Br}(K)$ is a countable abelian torsion group; hence, as an abstract group, it is completely determined by its Ulm invariants $U_{p}(\lambda, \operatorname{Br}(K))$, where $p$ is a prime number and $\lambda$ is an ordinal number (see the definition below). With this observation, Fein and Schacher initiated the investigation of the Ulm invariants of algebraic function fields over global fields, which culminated in the determination of all the Ulm invariants of the Brauer group of a rational function field $K$ in finitely many variables over a global field $k$ (see [FSS1]). For nonrational function fields in one variable over a global field $k$, the closest thing to a rational function field would be a nonrational function field $K$ of genus zero over $k$. In [FSS2], all the Ulm invariants $U_{p}(\lambda, \operatorname{Br}(K))$ for such a field $K$ are determined, except for one (!), namely $U_{2}(\omega 2, \operatorname{Br}(K))$. It turns out that all of the Ulm invariants of $\operatorname{Br}(K)$, except for the missing one, coincide with those of $\operatorname{Br}(k(t))$. The problem of this missing Ulm invariant has remained open; in fact, until now, there was not even a single example known of a nonrational genus zero function field over a global field for which the missing Ulm invariant $U_{2}(\omega 2, \operatorname{Br}(K))$ had been computed. In view of the fact that $U_{2}(\omega 2, \operatorname{Br}(k(t)))=0$, $\operatorname{Br}(K) \cong \operatorname{Br}(k(t))$ if and only if $U_{2}(\omega 2, \operatorname{Br}(K))=0$.

In this paper we determine the first known isomorphism classes of Brauer groups of nonrational genus zero extensions $E / k$ of number fields $k$. We prove the following

Main Theorem. Let $k$ be a totally real number field, and let $l / k$ be the quadratic subfield of the cyclotomic $\mathbb{Z}_{2}$-extension $k^{c y c} / k$.

Received by the editors February 14, 2003 and, in revised form, September 23, 2003.

2000 Mathematics Subject Classification. Primary 16K40, 12G05; Secondary 14H05.

The first author's research was supported by the Fund for Promotion of Research at the Technion.

The second author's research as supported in part by an International Research Fellowship, awarded by the National Science Foundation (INT-980199) and held at the Technion-Israel Institute of Technology during 1998-1999, and a Young Investigator Grant from the National Security Agency (MDA904-02-1-0061). 
Suppose that $E$ is a genus zero extension of $k$ which is split by $l$, and suppose that the Leopoldt Conjecture holds for the field $l$ and the prime 2. Then $\operatorname{Br}(E) \cong$ $\operatorname{Br}(k(t))$.

Since the Leopoldt Conjecture (see [NSW, §10.3]) is known to be true for all abelian number fields $[\mathrm{Br}$ (see also [NSW, Thms. 10.3.14 and 10.3.16]), we have

Corollary. Suppose $k$ is a totally real abelian number field not containing $\sqrt{2}$. Then if $E / k$ is a genus zero extension split by $k(\sqrt{2}), \operatorname{Br}(E) \cong \operatorname{Br}(k(t))$.

In particular, $\operatorname{Br}(E) \cong \operatorname{Br}(\mathbb{Q}(t))$ for all genus zero extensions $E$ of $\mathbb{Q}$ split by $\mathbb{Q}(\sqrt{2})$.

We approach $\operatorname{Br}(E)$ via $\operatorname{Br}(E l)$, where $l / k$ is a quadratic extension such that $\mathrm{El}$ is a rational function field. The Auslander-Brumer-Faddeev theorem establishes an isomorphism between $\operatorname{Br}(E l)$ and a direct sum of $\operatorname{Br}(l)$ and character groups of extensions of $l$, and we study an order two action on $\operatorname{Br}(E l)$ in terms of standard cohomological maps on these summands. Section 1 introduces this approach, and section 2 establishes properties of the order two action. Then in section 3 we present a technical analysis of heights of elements in the fixed subgroup of this order two action. Finally, in section 4 we prove the Main Theorem.

\section{Preliminaries}

Let $k$ be a perfect field. For any Galois field extension $K / k$, let $G_{K / k}$ denote the Galois group. Let $\bar{K}$ denote the algebraic closure of $K$ and $G_{K}$ the absolute Galois group $G_{\bar{K} / K}$.

We denote by $k^{*}$ the multiplicative group of $k$, by $\mathrm{X}(k)$ its character group $H^{1}\left(G_{k}, \mathbb{Q} / \mathbb{Z}\right)$, and by $\operatorname{Br}(k)$ its Brauer group $H^{2}\left(G_{k}, \bar{k}^{*}\right)$. Similarly, $\operatorname{Br}(K / k)$ denotes the relative Brauer group of $K / k$, identified with $H^{2}\left(G_{K / k}, K^{*}\right)$. All cohomology groups will be written additively, and all modules will be left modules.

For an additive abelian torsion group $A$, we write $A_{2}$ for the 2-primary component of $A$. The Ulm subgroups (for the prime 2) of $A_{2}$ are defined for any ordinary $\lambda$ by $A_{2}(0)=A_{2}, A_{2}(\lambda+1)=2 A_{2}(\lambda)$, and for $\lambda$ a limit ordinal, $A_{2}(\lambda)=\bigcap_{\lambda^{\prime}<\lambda} A_{2}\left(\lambda^{\prime}\right)$. The least $\lambda$ such that $A_{2}(\lambda)=A_{2}(\lambda+1)$ is the Ulm length of $A_{2}$. Now let $P(\lambda)=\left\{\alpha \in A_{2}(\lambda): 2 \alpha=0\right\}$. The Ulm invariant of $A_{2}$ at $\lambda$, denoted $U_{2}\left(\lambda, A_{2}\right)$, is $[P(\lambda) / P(\lambda+1): \mathbb{Z} / 2 \mathbb{Z}]$.

We write ht $A_{2}(\alpha)$ or ht $A(\alpha)$ for the height of $\alpha \in A_{2}$ in $A_{2}$, defined to be $\lambda$ such that $\alpha \in A_{2}(\lambda) \backslash A_{2}(\lambda+1)$ if such a $\lambda$ exists; otherwise, $\alpha \in A_{2}$ is divisible and we write $\mathrm{ht}_{A}(\alpha)=\infty$. We denote by $D(A)$ the divisible subgroup of $A$. For $\alpha \neq 0$, we will say that a divisible tower over $\alpha$ is a set $\left\{\alpha_{i}\right\}_{i=0}^{\infty}$ satisfying $2^{j} \alpha_{i}=\alpha_{i-j}$ for $j \leq i$ and $\alpha_{0}=\alpha$. Given nonzero $\alpha \in A_{2}, \alpha \in D\left(A_{2}\right)$ if and only if there exists a divisible tower over $\alpha$.

1.1. The genus zero extension $E$. A genus zero extension $E$ over a field $k$ is the quotient field of $k[x, y] /\left\langle 1-c x^{2}-d y^{2}\right\rangle$ for $c, d \in k^{*}$. We set $l=k(\sqrt{d})$. The quaternion algebra $(c, d)$ is split in $\operatorname{Br}(k)$ iff $E$ is isomorphic to a rational function field in one variable over $k$. We will assume throughout that $(c, d)$ is not split. Hence $l \not \subset E$.

Let $E l$ denote the compositum of $l$ and $E$. We determine an element $u$ such that $E l=l(u)$ as follows. Let $u=(1+\sqrt{d} y) / x$. Then $u(1-\sqrt{d} y) / x=c$, so that 
$c / u=(1-\sqrt{d} y) / x$. Moreover, $x=2 /(u+c / u)$ and $y=(2 u /(u+c / u)-1) / \sqrt{d}$, establishing that $E l=l(u)$.

We denote by $\sigma$ the unique nontrivial element of $G_{l / k}$ and by $s$ the nontrivial element of $G_{E l / E}$. We may extend $\sigma$ to an element of $G_{k}$, which by abuse of language we also denote by $\sigma$, and we similarly denote by $s$ the corresponding extension of $s$ to $G_{E \bar{k} / E}$.

In what follows $p$ will always be restricted to monic, irreducible $p \in l[u] \backslash\{u\}$, and for each $p$ we fix a root $a_{p}$ of $p$ for the remainder of the paper. If $\left\{a_{i}\right\} \subset \bar{k}^{*}$ are the roots of $p$, we set $\tilde{p} \in l[X]$ to be the monic, irreducible polynomial with roots $\left\{c / \sigma\left(a_{i}\right)\right\}$. Since $\sigma^{2} \in G_{l}, p \mapsto \tilde{p}$ is an order two action.

Lemma 1.1. The map s acts triangularly on

$$
\bar{k}(u)^{*}=\bar{k}^{*} \times\langle u\rangle \times \coprod_{a \in \bar{k}^{*}}\langle u+a\rangle
$$

by

(1) $s(a)=\sigma(a), a \in \bar{k}^{*}$;

(2) $s(u)=c \cdot \frac{1}{u}$; and

(3) $s(u+a)=\sigma(a) \cdot \frac{1}{u} \cdot\left(u+\frac{c}{\sigma(a)}\right), a \in \bar{k}^{*}$.

Moreover, for all $p, s(p)=\sigma(p(0)) \cdot u^{-\operatorname{deg} p} \cdot \tilde{p}(u)$.

For later use we define componentwise homomorphisms

$$
\begin{aligned}
& s_{u u}:\langle u\rangle \rightarrow\langle u\rangle, \\
& s_{u+a, u}:\langle u+a\rangle \rightarrow\langle u\rangle, \text { and } \\
& s_{p \tilde{p}}: \prod_{p(a)=0}\langle u+a\rangle \rightarrow \prod_{\tilde{p}(a)=0}\langle u+a\rangle
\end{aligned}
$$

by $s_{u u}\left(u^{e}\right)=s_{u+a, u}\left((u+a)^{e}\right)=1 / u^{e}$ and

$$
s_{p \tilde{p}}\left(\prod_{p(a)=0}(u+a)^{e_{a}}\right)=\prod_{p(a)=0}\left(u+\frac{c}{\sigma(a)}\right)^{e_{a}}=\prod_{\tilde{p}(a)=0}(u+a)^{e_{c / \sigma^{-1}(a)}} .
$$

1.2. The Brauer group of $E$. We have identified $\operatorname{Br}(E)$ with $H^{2}\left(G_{E}, \bar{E}^{*}\right)$. The algebraic closure $\bar{E}$ of $E$ is identical to the algebraic closure of $\bar{k}(u)$, and since by Tsen's theorem the Brauer group of $\bar{k}(u)$ is trivial, we have that $H^{2}\left(G_{E}, \bar{E}^{*}\right) \cong$ $H^{2}\left(G_{\bar{k}(u) / E}, \bar{k}(u)^{*}\right)$. Now every element $\gamma \in G_{k}$ lifts to $\gamma^{\prime} \in G_{\bar{k}(u) / E}$ by extending the automorphism trivially on $x$ and $y$. Inversely, since $\bar{k}(u)=E \otimes_{k} \bar{k}$ and $k$ is algebraically closed in $E$, any element $\gamma^{\prime} \in G_{\bar{k}(u) / E}$ sends $\bar{k}$ to $\bar{k}$. Therefore we may and do identify $G_{\bar{k}(u) / E}$ with $G_{k}$, and we have $\operatorname{Br}(E) \cong H^{2}\left(G_{k}, \bar{k}(u)^{*}\right)$.

Now by Hilbert's Theorem $90, H^{1}\left(G_{l}, \bar{k}(u)^{*}\right)$ is trivial. Moreover, since $G_{l / k}$ is finite cyclic, $H^{3}\left(G_{l / k}, l(u)^{*}\right) \cong H^{1}\left(G_{l / k}, l(u)^{*}\right)$, which is also trivial by Theorem 90. The standard inflation-restriction five-term exact sequence [NSW Prop. 1.6.6] beginning with $H^{2}$ then begins

$$
0 \rightarrow H^{2}\left(G_{l / k}, l(u)^{*}\right) \rightarrow H^{2}\left(G_{k}, \bar{k}(u)^{*}\right) \stackrel{\phi}{\rightarrow} H^{2}\left(G_{l}, \bar{k}(u)^{*}\right)^{G_{l / k}} \rightarrow 0,
$$

or, equivalently,

$$
0 \rightarrow \operatorname{Br}(E l / E) \rightarrow \operatorname{Br}(E) \rightarrow \operatorname{Br}(l(u))^{G_{l / k}} \rightarrow 0
$$


Now $\sigma \in G_{k}$ acts naturally on $\operatorname{Br}(l(u))=H^{2}\left(G_{l}, \bar{k}(u)^{*}\right)$ : given a 2-cocycle $h$, this action is $h^{s}\left(g_{1}, g_{2}\right)=s\left(h\left(\sigma^{-1} g_{1} \sigma, \sigma^{-1} g_{2} \sigma\right)\right)$, where $s$ acts on $\bar{k}(u)^{*}$ as above. We denote this action on $\operatorname{Br}(l(u))$ by $s^{*}$, and the fixed group in (1.1) on the right is therefore $\operatorname{Br}(l(u))^{\left\langle s^{*}\right\rangle}$.

1.3. The Auslander-Brumer-Faddeev decomposition. Recall that the Auslander-Brumer-Faddeev theorem ([AB, Prop. 4.1], [Fa, Thms. 15.2, 15.3]) establishes an isomorphism

$$
\operatorname{Br}(l(u)) \cong \operatorname{Br}(l) \oplus \mathrm{X}(l) \oplus\left(\bigoplus_{p} \mathrm{X}\left(l\left(a_{p}\right)\right)\right) .
$$

We will need the particular isomorphisms contained in the proof of this result, which we review as follows.

Let $G=G_{l}$ and for an arbitrary fixed $p, H=G_{l\left(a_{p}\right)}$. Let $A=\operatorname{Ind}_{G}^{H}(\mathbb{Z})=$ $\operatorname{Hom}_{H}(G, \mathbb{Z})$ be the $G$-module of $H$-module homomorphisms from $G$ to the trivial $G$-module $\mathbb{Z} ; g \in G$ acts on $x \in \operatorname{Ind}_{G}^{H}(\mathbb{Z})$ via $(g x)\left(g_{1}\right)=x\left(g_{1} g\right)$. $A$ may be considered the set of functions from $G$ to $\mathbb{Z}$ defined on right cosets of $H$ in $G$. Define $\bar{x}\left(g_{1}\right)=x\left(g_{1}^{-1}\right)$ for $x \in A$. Then $\bar{x}$ is defined on left cosets of $H$ in $G$ and $\overline{g x}\left(g_{1}\right)=\bar{x}\left(g^{-1} g_{1}\right)$.

Consider $B=B(p):=\prod_{p(a)=0}\langle u-a\rangle \subset \bar{k}(u)^{*}$. We claim that $A$ and $B$ are isomorphic $G$-modules under the map $\iota: A \rightarrow B$ given by

$$
\iota(x)=\prod_{\tau H \in G / H}\left(u-\tau\left(a_{p}\right)\right)^{\bar{x}(\tau)} .
$$

To check that $\iota$ respects $G$-action, we calculate

$$
\begin{aligned}
\iota(g x) & =\prod_{\tau H \in G / H}\left(u-\tau\left(a_{p}\right)\right)^{\overline{g x}(\tau)}=\prod_{\tau H \in G / H}\left(u-\tau\left(a_{p}\right)\right)^{\bar{x}\left(g^{-1} \tau\right)} \\
& =\prod_{g \tau H \in G / H}\left(u-g \tau\left(a_{p}\right)\right)^{\bar{x}(\tau)}=g\left(\prod_{\tau H \in G / H}\left(u-\tau\left(a_{p}\right)\right)^{\bar{x}(\tau)}\right)=g \iota(x) .
\end{aligned}
$$

The proof of the Auslander-Brumer-Faddeev theorem proceeds by splitting the $G_{l}$-module $\bar{k}(u)^{*}$ of $H^{2}\left(G_{l}, \bar{k}(u)^{*}\right)$ as

$$
\bar{k}(u)^{*}=\bar{k}^{*} \times\langle u\rangle \times \coprod_{p} \prod_{p(a)=0}\langle u-a\rangle,
$$

and then realizing the summands $\mathrm{X}(l)$ and $\mathrm{X}\left(l\left(a_{p}\right)\right)$ with the isomorphisms

$$
\mathrm{X}(l)=H^{1}\left(G_{l}, \mathbb{Q} / \mathbb{Z}\right) \stackrel{\delta}{\rightarrow} H^{2}\left(G_{l}, \mathbb{Z}\right) \stackrel{\pi^{*}}{\rightarrow} H^{2}\left(G_{l},\langle u\rangle\right)
$$

and

$$
\mathrm{X}\left(l\left(a_{p}\right)\right)=H^{1}(H, \mathbb{Q} / \mathbb{Z}) \stackrel{\delta}{\rightarrow} H^{2}(H, \mathbb{Z}) \stackrel{s h}{\longrightarrow} H^{2}(G, A) \stackrel{\iota^{*}}{\longrightarrow} H^{2}(G, B) .
$$

Here $\delta$ denotes the standard coboundary map, sh the map of Shapiro's Lemma, and $\pi: \mathbb{Z} \rightarrow\langle u\rangle$ the homomorphism $\pi(e)=u^{e}$ of trivial $G_{l}$-modules $\mathbb{Z}$ and $\langle u\rangle$.

We identify $\operatorname{Br}(l(u))$ with the decomposition (1.2) and denote an arbitrary element of this group by $\beta+\chi_{u}+\sum \chi_{p}$ or $\beta \oplus \chi_{u} \oplus \sum \chi_{p}$.

By Lemma 1.1. since $s$ acts triangularly on $\bar{k}(u)^{*}$ on the factors in (1.3) and $s_{p \tilde{p}}$ sends $B(p)$ to $B(\tilde{p})$, we have the following. 
Lemma 1.2. The map $s^{*}$ acts triangularly on $\operatorname{Br}(l(u))$. In particular, for arbitrary $\beta \oplus \chi_{u} \oplus \sum \chi_{p} \in \operatorname{Br}(l(u))$,

$$
\begin{aligned}
s^{*}\left(\beta \oplus \chi_{u} \oplus \sum \chi_{p}\right)= & \left(s_{11}^{*}(\beta)+s_{u 1}^{*}\left(\chi_{u}\right)+\sum s_{p 1}^{*}\left(\chi_{p}\right)\right) \\
& \oplus\left(s_{u u}^{*}\left(\chi_{u}\right)+\sum s_{p u}^{*}\left(\chi_{p}\right)\right) \\
& \oplus \sum s_{p \tilde{p}}\left(\chi_{p}\right),
\end{aligned}
$$

where we denote the component parts of $s^{*}$ as follows:

(a) $s_{p \tilde{p}}^{*}: \mathrm{X}\left(l\left(a_{p}\right)\right) \rightarrow \mathrm{X}\left(l\left(a_{\tilde{p}}\right)\right)$;

(b) $s_{p u}^{*}: \mathrm{X}\left(l\left(a_{p}\right)\right) \rightarrow \mathrm{X}(l)$;

(c) $s_{u u}^{*}: \mathrm{X}(l) \rightarrow \mathrm{X}(l)$;

(d) $s_{p 1}^{*}: \mathrm{X}\left(l\left(a_{p}\right)\right) \rightarrow \operatorname{Br}(l)$;

(e) $s_{u 1}^{*}: \mathrm{X}(l) \rightarrow \operatorname{Br}(l)$; and

(f) $s_{11}^{*}: \operatorname{Br}(l) \rightarrow \operatorname{Br}(l)$.

For later use we further define $s_{u}^{*}=\sum_{p} s_{p u}^{*}$ and $s_{1}^{*}=s_{u 1}^{*}+\sum_{p} s_{p 1}^{*}$.

\section{Decomposing $s^{*}$ on $\operatorname{Br}(l(u))$}

In this section we study the component functions of $s^{*}$, comparing them to natural Galois actions on $\operatorname{Br}(l)$ and $\mathrm{X}(l)$. We denote by $\sigma$ the natural Galois action of $\sigma$ on $\operatorname{Br}(l) \cong H^{2}\left(G_{l}, \bar{k}^{*}\right)$ and on $\mathrm{X}(l) \cong H^{1}\left(G_{l}, \mathbb{Q} / \mathbb{Z}\right)$. Note that in $H^{1}\left(G_{l}, \mathbb{Q} / \mathbb{Z}\right)$, $\mathbb{Q} / \mathbb{Z}$ is a trivial $G_{k}$-module.

2.1. $s_{11}^{*}$ on $\operatorname{Br}(l)$ and $\operatorname{Br}\left(l_{\mathfrak{p}}\right)$. Since $s$ acts on $\bar{k}^{*}$ via $\sigma$, the action of $s_{11}^{*}$ on $\operatorname{Br}(l)$ is the natural Galois action

$$
s_{11}^{*}(\beta)=\sigma(\beta), \quad \beta \in \operatorname{Br}(l) .
$$

We now determine the action of $\sigma$ on the local invariants $b_{\mathfrak{p}}$ of an element $b$ of $\operatorname{Br}(l)$ with the following general result.

Proposition 1. Let $K / k$ be a finite Galois extension of global fields. Suppose $g \in G_{K / k}$ and $\mathfrak{p}$ is a prime of $K$, and let $b_{\mathfrak{p}}$ denote the local invariant of $b \in \operatorname{Br}(K)$ at $\mathfrak{p}$. Then

$$
(g(b))_{\mathfrak{p}}=b_{g^{-1}(\mathfrak{p})} .
$$

Proof. For an arbitrary field $K$, a $K$-algebra $A$ is an associative ring $A$ with unity together with an embedding $\alpha: K \hookrightarrow A$ of $K$ into the center of $A$. Thus a $K$ algebra should be considered a pair $(A, \alpha)$. Now let $(B, \beta)$ be a second $K$-algebra. Then the tensor product

$$
(C, \gamma)=(A, \alpha) \otimes_{K}(B, \beta)
$$

is generated by elements $a \otimes b, a \in A, b \in B$, satisfying $\alpha(r) a \otimes b=a \otimes \beta(r) b$, $r \in K$, and where $\gamma=\alpha \odot \beta: K \hookrightarrow C$ is defined by $\gamma(r)=\alpha(r) \otimes 1_{B}=1_{A} \otimes \beta(r)$.

Let $g$ be an automorphism of $K$. An action of $g$ on $(A, \alpha)$ may be defined by $(A, \alpha)^{g}:=\left(A, \alpha^{g}\right)$, where $\alpha^{g}(r)=\alpha g(r)$ for $r \in K$. Note that as rings, $(A, \alpha)$ and $(A, \alpha)^{g}$ are isomorphic. It follows that $\gamma^{g}(r)=\gamma g(r)=\alpha g(r) \otimes 1=1 \otimes \beta g(r)$, i.e., $\alpha^{g}(r) \otimes 1=1 \otimes \beta^{g}(r)$, so

$$
\gamma^{g}=(\alpha \odot \beta)^{g}=\alpha^{g} \odot \beta^{g} .
$$


We now specialize to the case where $(A, \alpha)$ is a finite-dimensional central simple $K$-algebra and $B$ is a complete discrete valued field $K_{\mathfrak{p}}$, where $\mathfrak{p}$ will denote the valuation of $K_{\mathfrak{p}}$ as well as the valuation induced on $K$ by the embedding $\beta$ of $K$ into $K_{\mathfrak{p}}$. By (2.2),

$$
\left(\alpha^{g^{-1}} \odot \beta\right)^{g}=\alpha \odot \beta^{g} .
$$

Note again that, as rings, $\left(C,\left(\alpha^{g^{-1}} \odot \beta\right)^{g}\right)$ and $\left(C, \alpha^{g^{-1}} \odot \beta\right)$ are isomorphic. Moreover, $\left(C,\left(\alpha^{g^{-1}} \odot \beta\right)^{g}\right)$ and $\left(C, \alpha \odot \beta^{g}\right)$ both represent finite-dimensional central simple algebras over complete discrete valued fields.

Now assume the hypotheses of the proposition. We use a lemma of Janusz Ja. Lemma, p. 385], which says the following: let $L_{1}$ and $L_{2}$ be finite extensions of $\mathbb{Q}_{p}$ and let $A_{i}$ be central simple $L_{i}$-algebras, $i=1,2$. If $A_{1}$ and $A_{2}$ are isomorphic as rings, then $\operatorname{inv}\left(A_{1}\right)=\operatorname{inv}\left(A_{2}\right)$. By Janusz' lemma, $\left(C,\left(\alpha^{g^{-1}} \odot \beta\right)^{g}\right)$ and $\left(C, \alpha^{g^{-1}} \odot\right.$ $\beta$ ) have the same local invariant. It follows that $\left(C, \alpha^{g^{-1}} \odot \beta\right)$ and $\left(C, \alpha \odot \beta^{g}\right)$ have the same local invariant.

Remark. In the proof of Janusz' lemma, the assertion is made that a field isomorphism of $p$-adic local fields is an isomorphism of $p$-adic local fields, i.e., that prime elements are mapped to prime elements. To prove this statement, the essential idea is the same as in the proof that the only algebraic automorphism of $\mathbb{Q}_{p}$ is the identity. Suppose $L_{i}, i=1,2$, are finite extensions of $\mathbb{Q}_{p}$ with ramification indices $e_{i}$ and normalized valuations $v_{i}, f: L_{1} \rightarrow L_{2}$ is a field isomorphism, and $\pi$ is a prime element of $L_{1}$. Then $\pi^{e_{1}}=p u$ with $u \in L_{1}$ a unit. Write $f(\pi)^{e_{1}}=f(p u)=f(p) f(u)=p f(u)$. Now if $f(u)$ is a unit in $L_{2}$, we are done, since $e_{1} v_{2}(f(\pi))=v_{2}(p)=e_{2}, e_{1} \mid e_{2}$ and by symmetry $e_{1}=e_{2}$ and $v_{2}(f(\pi))=1$. That $f$ maps units to units follows from the fact that the units of a $p$-adic field may be characterized algebraically, as the only elements that have $n$th roots in the field for infinitely many $n$.

2.2. $s_{u u}^{*}$ on $\mathbf{X}(l)$.

Proposition 2. In the decomposition (1.2),

$$
s_{u u}^{*}(\chi)=-\sigma(\chi), \quad \chi \in \mathrm{X}(l),
$$

where $\sigma$ denotes the natural Galois action on $\mathrm{X}(l)=H^{1}\left(G_{l}, \mathbb{Q} / \mathbb{Z}\right)$.

Proof. The homomorphism $s_{u u}^{*}$ acts on a 2-cocycle $f \in H^{2}\left(G_{l},\langle u\rangle\right)$ by

$$
f\left(g_{1}, g_{2}\right) \mapsto s_{u u}\left(f\left(\sigma^{-1} g_{1} \sigma, \sigma^{-1} g_{2} \sigma\right)\right)=-f\left(\sigma^{-1} g_{1} \sigma, \sigma^{-1} g_{2} \sigma\right),
$$

since the image of $f$ lies in $\langle u\rangle$ and $s_{u u}$ is the inversion map. Now $\mathrm{X}(l)$ appears in (1.2) under the isomorphisms in (1.4). Using the facts that in the natural Galois actions on $H^{1}\left(G_{l}, \mathbb{Q} / \mathbb{Z}\right)$ and $H^{2}\left(G_{l}, \mathbb{Z}\right)$, the action of $\sigma$ on the modules $\mathbb{Q} / \mathbb{Z}$ and $\mathbb{Z}$ is trivial, and that $\sigma$ commutes with coboundary maps $\delta$ [NSW Prop. 1.5.2], a direct calculation yields

$$
\delta^{-1} \circ\left(\pi^{*}\right)^{-1} \circ s_{u u}^{*} \circ \pi^{*} \circ \delta=-\sigma .
$$


2.3. $s_{p u}^{*}$ on $\mathbf{X}\left(l\left(a_{p}\right)\right)$.

Proposition 3. In the decomposition (1.2),

$$
s_{p u}^{*}(\chi)=-\sigma\left(\operatorname{Cor}_{l(a) / l}\left(\chi_{p}\right)\right), \quad \chi_{p} \in \mathrm{X}\left(l\left(a_{p}\right)\right),
$$

where $\sigma$ denotes the natural Galois action on $\mathrm{X}(l)=H^{1}\left(G_{l}, \mathbb{Q} / \mathbb{Z}\right)$.

Proof. Let $B=\prod_{p(a)=0}\langle u-a\rangle$. The homomorphism $s_{p u}^{*}$ acts on a 2-cocycle $f \in$ $Z^{2}\left(G_{l}, B\right)$ by

$$
f\left(g_{1}, g_{2}\right) \mapsto\left(\prod s_{u+a, u}\right)\left(f\left(\sigma^{-1} g_{1} \sigma, \sigma^{-1} g_{2} \sigma\right)\right),
$$

where for each $a, s_{u+a, u}(u+a)=\frac{1}{u}$. Further let $\rho: B \rightarrow\langle u\rangle$ be defined by

$$
\rho\left(\prod_{p(a)=0}(u-a)^{e_{a}}\right)=u^{\left(\sum_{p(a)=0} e_{a}\right)}
$$

and let $\rho^{*}$ be the induced map $\rho^{*}: H^{2}\left(G_{l}, B\right) \rightarrow H^{2}\left(G_{l},\langle u\rangle\right)$ of cohomology. Observe that $s_{p u}=s_{u u} \circ \rho$, hence $s_{p u}^{*}=s_{u u}^{*} \circ \rho^{*}$.

Keeping the notation for $G, H$, and $A$ as in (1.5), define a $G$-module homomorphism $\nu: A \rightarrow \mathbb{Z}$ by

$$
\nu(x)=\sum_{\tau H \in G / H} \tau x\left(\tau^{-1}\right)=\sum_{\tau H \in G / H} x\left(\tau^{-1}\right) .
$$

We calculate

$$
\begin{aligned}
\rho(\iota(x)) & =\rho\left(\prod_{\tau H \in G / H}\left(u-\tau\left(a_{p}\right)\right)^{\bar{x}(\tau)}\right)=\prod_{\tau H \in G / H} u^{\bar{x}(\tau)} \\
& =u^{\left(\sum_{\tau H \in G / H} x\left(\tau^{-1}\right)\right)}=\pi\left(\sum_{\tau H \in G / H} x\left(\tau^{-1}\right)\right)=\pi(\nu(x)) .
\end{aligned}
$$

Hence we have a commutative diagram:

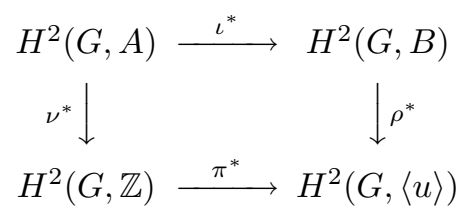

Now $\nu^{*} \circ s h$ is the corestriction map from $H^{2}\left(G_{l\left(a_{p}\right)}, \mathbb{Z}\right) \rightarrow H^{2}\left(G_{l}, \mathbb{Z}\right)$ NSW Prop. 1.6.4]. Moreover, the coboundary map $\delta$ from $H^{1}$ to $H^{2}$ commutes with the corestriction map [NSW, Prop. 1.5.2]. Hence under the isomorphisms in (1.4) and (1.5),

$$
\begin{aligned}
\delta^{-1} \circ\left(\pi^{*}\right)^{-1} \circ \rho^{*} \circ \iota^{*} \circ s h \circ \delta & =\delta^{-1} \circ \nu^{*} \circ s h \circ \delta \\
& =\delta^{-1} \circ\left(\operatorname{Cor}_{H^{2}}\right) \circ \delta=\operatorname{Cor}_{H^{1}} .
\end{aligned}
$$

Therefore in the decomposition (1.2), $\rho^{*}$ is the corestriction map, and since $s_{p u}^{*}=$ $s_{u u}^{*} \circ \rho^{*}$, by (2.3),$s_{p u}^{*}=-\sigma \operatorname{Cor}_{l(a) / l}$. 
2.4. $s_{p \tilde{p}}^{*}$ on $\mathrm{X}\left(l\left(a_{p}\right)\right)$. Let Cor: $\bigoplus \mathrm{X}\left(l\left(a_{p}\right)\right) \rightarrow \mathrm{X}(l)$ be the sum $\sum \operatorname{Cor}_{l\left(a_{p}\right) / l}$ of the corestrictions on each summand.

Proposition 4. In the decomposition (1.2),

$$
\operatorname{Cor}_{l\left(a_{\tilde{p}}\right) / l}\left(s_{p \tilde{p}}^{*}\left(\chi_{p}\right)\right)=\sigma\left(\operatorname{Cor}_{l(a) / l}\left(\chi_{p}\right)\right), \quad \chi_{p} \in \mathrm{X}\left(l\left(a_{p}\right)\right),
$$

and if $p=\tilde{p}$, then

$$
s_{p p}^{*}\left(\chi_{p}\right)=\tilde{\sigma}\left(\chi_{p}\right), \quad \chi_{p} \in \mathrm{X}\left(l\left(a_{p}\right)\right),
$$

where $\tilde{\sigma}=\sigma \tau$ for a $\tau \in G_{l}$ such that $\tilde{\sigma}\left(a_{p}\right)=c / a_{p}$.

If for all $p, s_{p \tilde{p}}^{*}\left(\chi_{p}\right)=\chi_{\tilde{p}}$, then

$$
\sigma\left(\operatorname{Cor} \sum \chi_{p}\right)=\operatorname{Cor} \sum \chi_{p}
$$

and if additionally $\chi_{u} \in \mathrm{X}(l)$ such that $(1+\sigma) \chi_{u}=-\operatorname{Cor} \sum \chi_{p}$, then

$$
\sigma\left(s_{1}^{*}\left(\chi_{u}+\sum \chi_{p}\right)\right)=-s_{1}^{*}\left(\chi_{u}+\sum \chi_{p}\right) .
$$

Proof. Equations (2.5), (2.7), and (2.8) each follow from the fact that $s^{*}$ has order 2 . For the first result, let $\chi_{p} \in \mathrm{X}\left(l\left(a_{p}\right)\right)$ be arbitrary and set $\chi_{\tilde{p}}=s_{p \tilde{p}}^{*}\left(\chi_{p}\right)$. Then by applying (1.6) twice, we see that the component of $s^{*}\left(s^{*}\left(\chi_{p}\right)\right)=\chi_{p}$ in $\mathrm{X}(l)$ is

$$
s_{u u}^{*} s_{p u}^{*}\left(\chi_{p}\right)+s_{\tilde{p} u}^{*}\left(\chi_{\tilde{p}}\right),
$$

which, by (2.3) and (2.4), is Cor $\chi_{p}-\sigma$ Cor $\chi_{\tilde{p}}$. Then since this component must be trivial, the first result follows. A similar argument establishes (2.7) and (2.8).

For (2.6), first observe that if $p=\tilde{p}$, then the action $a \mapsto c / \sigma(a)$ permutes the roots of $p$. Hence $c / \sigma(a)=a_{p}$ for some root $a$. Since $G_{l}$ is transitive on the roots of $p, a=\tau\left(a_{p}\right)$ for some $\tau \in G_{l}$. Hence $a_{p}=c / \sigma \tau\left(a_{p}\right)$. Let $\tilde{\sigma}=\sigma \tau$.

Keeping the notation for $G, H, A$, and $B$ as in (1.5), observe that $s_{p p}^{*}$ acts on $H^{2}(G, B)$ by sending an arbitrary 2 -cocycle $f\left(g_{1}, g_{2}\right)$ to

$$
s_{p p}\left(f\left(\sigma^{-1} g_{1} \sigma, \sigma^{-1} g_{2} \sigma\right)\right) \text {. }
$$

Now $\tau^{*}$ acts on $H^{2}(G, B)$ trivially [NSW] Prop. 1.6.2], so $s_{p p}^{*} \circ \tau^{*}=s_{p p}^{*}$. However, direct calculation shows that $s_{p p}^{*} \circ \tau^{*}$ sends $f\left(g_{1}, g_{2}\right)$ to

$$
\left(s_{p p} \circ \tau\right)\left(f\left(\tilde{\sigma}^{-1} g_{1} \tilde{\sigma}, \tilde{\sigma}^{-1} g_{2} \tilde{\sigma}\right)\right),
$$

where

$$
\begin{aligned}
\left(s_{p p} \circ \tau\right)\left(\prod_{p(a)=0}(u-a)^{e_{a}}\right) & =\prod\left(u-\frac{c}{\sigma \tau(a)}\right)^{e_{a}} \\
& =\left(u-a_{p}\right)^{e_{a_{p}}} \prod_{a \neq a_{p}}\left(u-\frac{c}{\sigma \tau(a)}\right)^{e_{a}} .
\end{aligned}
$$

Hence $s_{p p} \circ \tau$ acts trivially on $\left\langle u-a_{p}\right\rangle \subset B$, and consequently $\iota^{-1} \circ\left(s_{p p} \circ \tau\right) \circ \iota$ acts trivially on the values $x(1), x \in A$.

Recall that $(s h)^{-1}$ is induced by the module map $A \rightarrow \mathbb{Z}$ given by $x \mapsto x(1)=$ $x(H)$ [NSW, Prop. 1.6.3]; hence $(s h)^{-1} \circ\left(\iota^{*}\right)^{-1} \circ s_{p p}^{*} \circ \tau^{*} \circ \iota^{*} \circ s h$ acts on $H^{2}(H, \mathbb{Z})$ by sending a 2 -cocycle $f\left(g_{1}, g_{2}\right)$ to $f\left(\tilde{\sigma}^{-1} g_{1} \tilde{\sigma}, \tilde{\sigma}^{-1} g_{2} \tilde{\sigma}\right)$. As a result,

$$
\tilde{\sigma}=s h^{-1} \circ\left(\iota^{*}\right)^{-1} \circ s_{p p}^{*} \circ \iota^{*} \circ s h
$$

and since $\tilde{\sigma}$ commutes with coboundary maps [NSW, Prop. 1.5.2], $s_{p p}^{*}\left(\chi_{p}\right)=\tilde{\sigma}\left(\chi_{p}\right)$. 


\section{The FIXED SubGroup $\operatorname{Br}(l(u))^{\left\langle s^{*}\right\rangle}$}

3.1. Notation. Let $\mathrm{B}^{G}$ denote the fixed subgroup $\operatorname{Br}(l(u))_{2}^{\left\langle s^{*}\right\rangle}$ of $\operatorname{Br}(l(u))_{2}$. By (1.6) and (2.7), a direct calculation shows that this group consists of precisely those elements satisfying

(1) $s_{p \tilde{p}}^{*} \chi_{p}=\chi_{\tilde{p}}, \forall p$

(2) $(1+\sigma) \chi_{u}=-\operatorname{Cor} \sum \chi_{p} ;$ and

(3) $(1-\sigma) \beta=s_{1}^{*}\left(\chi_{u}+\sum \chi_{p}\right)$.

Let $\mathrm{X}_{\neq u}=\bigoplus_{p} \mathrm{X}\left(l\left(a_{p}\right)\right)_{2}$ and $\mathrm{X}=\mathrm{X}(l)_{2} \oplus \mathrm{X}_{\neq u}$. We further define $\mathrm{X}_{\neq u}^{G}$ and $\mathrm{X}^{G}$ as follows:

$$
\begin{aligned}
\mathrm{X}_{\neq u}^{G}= & \left\{\sum \chi_{p} \in \mathrm{X}_{\neq u}: s_{p \tilde{p}}^{*}\left(\chi_{p}\right)=\chi_{\tilde{p}} \forall p\right\} \\
\mathrm{X}^{G}= & \left\{\chi=\chi_{u}+\sum \chi_{p} \in \mathrm{X}:(1+\sigma) \chi_{u}=-\operatorname{Cor} \sum \chi_{p},\right. \\
& \left.s_{p \tilde{p}}^{*} \chi_{p}=\chi_{\tilde{p}} \forall p\right\} .
\end{aligned}
$$

Now assume that $k$, and so also $l$, is a number field. Then $D\left(\mathrm{X}(l)_{2}\right)$ is of finite rank [NSW, Thm. 11.1.2], where as usual $D(A)$ denotes the divisible subgroup of $A$, and its dual $\operatorname{Hom}\left(D\left(\mathrm{X}(l)_{2}\right), \mathbb{Q} / \mathbb{Z}\right)$ is a free $\mathbb{Z}_{2}$-module of finite rank. Applying CR, Thm. 74.3] and passing to the dual again, we may then decompose $D\left(\mathrm{X}(l)_{2}\right)$ as

$$
D\left(\mathrm{X}(l)_{2}\right)=I \oplus N \oplus P,
$$

where each of $I, N$, and $P$ is a finite direct sum of $\mathbb{Q}_{2} / \mathbb{Z}_{2}$ summands and the natural Galois action $\sigma$ is trivial on $I$, negation on $N$, and permutation of pairs of $\mathbb{Q}_{2} / \mathbb{Z}_{2}$ summands on $P$. Then $D\left(\mathrm{X}(l)_{2}\right)^{\langle\sigma\rangle}=I \oplus{ }_{2} N \oplus P^{\langle\sigma\rangle}$.

Remark. A sketch of an elementary proof of (3.1) is as follows. Let $M$ be a direct sum of finitely many copies of $\mathbb{Q}_{2} / \mathbb{Z}_{2}$, and let $\sigma$ be an order 2 action on $M$. Denote by $V$ the $\mathbb{F}_{2}$-vector space given by the 2-torsion ${ }_{2} M$ of $M$. By linear algebra $V$ decomposes into $V_{I} \oplus V_{P} \oplus \sigma\left(V_{P}\right)$. Let $W_{1}$ be a complement of $\left(V_{P}+\sigma\left(V_{P}\right)\right) \cap$ $(1+\sigma) M$ in $V \cap(1+\sigma) M$ and $W_{-1}$ a complement of $\left(V_{P}+\sigma\left(V_{P}\right)\right) \cap(1-\sigma) M$ in $V \cap(1-\sigma) M$. Using the fact that $M=2 M=(1+\sigma) M+(1-\sigma) M$, one shows that $V=V_{1} \oplus V_{-1} \oplus V_{P} \oplus \sigma\left(V_{P}\right)$. Now since $M$ is divisible, the homomorphic image $(1+\sigma) M$ is divisible; we may therefore construct a divisible tower inside $(1+\sigma) M$ over each element of a basis of $W_{1}$. Denote by $I$ the subgroup generated by these towers. Similarly denote by $N$ the subgroup generated by towers inside $(1-\sigma) M$ constructed over each element of a basis of $W_{-1}$. Finally construct towers over each basis element of $V_{P}$ and let $P$ be the subgroup generated by these towers and their $\sigma$-conjugates. Observe that a sum $\sum M_{i}$ of subgroups $M_{i}$ of $M$ is direct if and only if $\sum\left(M_{i} \cap V\right)=\bigoplus\left(M_{i} \cap V\right)$. Moreover, if each $M_{i}$ is divisible and $\sum \operatorname{dim}_{\mathbb{F}_{2}}\left(M_{i} \cap V\right)=\operatorname{dim}_{\mathbb{F}_{2}} V$, then $M=\bigoplus M_{i}$. Hence $M=I \oplus N \oplus P$.

Each $w \in D\left(\mathrm{X}(l)_{2}\right)^{\langle\sigma\rangle}$ of order 2 represents a cyclic extension of $l$ of degree 2 which is fixed under the action of $\sigma$. By Kummer theory $w=l(\sqrt{e})$ for $e \in l^{*} \backslash l^{* 2}$, and since $w$ is fixed by $\sigma, l(\sqrt{e})$ is Galois over $k$. The group $G_{l(\sqrt{e}) / k}$ is isomorphic either to $\mathbb{Z} / 4 \mathbb{Z}$ or to $\mathbb{Z} / 2 \mathbb{Z} \oplus \mathbb{Z} / 2 \mathbb{Z}$. One may choose a representative in $k^{*}$ for the class of $e$ in $l^{*} / l^{* 2}$ if and only if $G_{l(\sqrt{e}) / k} \cong \mathbb{Z} / 2 \mathbb{Z} \oplus \mathbb{Z} / 2 \mathbb{Z}$. In this case we write $w=l(\sqrt{e}), e \in k^{*} \backslash k^{* 2}$. We also write that $w=0$ satisfies $w=l(\sqrt{1})$. 
Define $W$ to be the exponent 2 subgroup of $D\left(\mathrm{X}(l)_{2}\right)^{\langle\sigma\rangle}$ consisting of extensions with Klein 4-group over $k$, together with the identity element, as follows:

$$
W=\left\{w \in D\left(\mathrm{X}(l)_{2}\right)^{\langle\sigma\rangle}:|w| \leq 2, w=l(\sqrt{e}), e \in k^{*}\right\} .
$$

For an element $w \in D\left(\mathrm{X}(l)_{2}\right)$, write $w_{I}$ for the component of $w$ in $I$, and set $W_{I}=\left\{w_{I} \in I: w \in W\right\}$.

Now define a function $\lambda$ from $W_{I}$ to the set of ordinals as follows:

$$
\lambda_{w}=\sup \left\{\operatorname{ht}_{\mathrm{X}_{\neq u}^{G}}\left(\sum \chi_{p}\right):\left|\sum \chi_{p}\right|=2, \quad\left(\operatorname{Cor} \sum \chi_{p}\right)_{I}=w\right\} .
$$

We will repeatedly use the fact that the Ulm length of $\mathrm{X}(K)$ for $K$ a number field is less than $\omega 2\left[\mathrm{FS}\right.$, Thm. 1], and hence that the Ulm lengths of $\mathrm{X}_{\neq u}$ and $\mathrm{X}$ are at most $\omega 2$. Here, this result implies that for any $w \in W_{I}$ we have that $\lambda_{w} \leq \omega 2$ or $\lambda_{w}=\infty$.

\subsection{Reducing to $\mathbf{X}^{G}$.}

Proposition 5. Suppose $k$ is a number field. Then there exists an order 2 element in $\mathrm{B}^{G}$ of height $\omega 2$ if and only if there exists an order 2 element in $\mathrm{X}^{G}$ of height $\omega 2$.

Lemma 3.1. Suppose $k$ is a number field and that $\alpha=\beta+\chi \in \mathrm{B}^{G}$ has $\beta \in D(\operatorname{Br}(l))$ and $\mathrm{ht}_{\mathrm{X}^{G}}(\chi) \geq i+1$ for some $i \in \mathbb{N}_{\geq 0}$. Then $\mathrm{ht}_{\mathrm{B}^{G}}(\alpha) \geq i$.

Proof. We are given that there exists $\chi_{i+1} \in \mathrm{X}^{G}$ with $2^{i+1} \chi_{i+1}=\chi$. Let $\chi_{i}=$ $2 \chi_{i+1}$. By specifying the element by invariants at each place $\mathfrak{p}$ of $l$, we will construct $\beta_{i} \in \operatorname{Br}(l)$ such that $2^{i} \beta_{i}=\beta$ and $(1-\sigma) \beta_{i}=s_{1}^{*} \chi_{i}$. Then $\alpha_{i}=\beta_{i}+\chi_{i}$ satisfies $\alpha_{i} \in \mathrm{B}^{G}$ and $2^{i} \alpha_{i}=\alpha$.

Consider places $\mathfrak{p}_{1}, \mathfrak{p}_{2}$ permuted by $\sigma$. Let $x=\beta_{\mathfrak{p}_{1}}, y=\beta_{\mathfrak{p}_{2}}$. Since $\alpha \in \mathrm{B}^{G}$, $(1-\sigma) \beta=s_{1}^{*} \chi$. Then, by (2.1),$\left(s_{1}^{*} \chi\right)_{\mathfrak{p}_{1}}=((1-\sigma) \beta)_{\mathfrak{p}_{1}}=x-y$. Let $z=\left(s_{1}^{*} \chi_{i+1}\right)_{\mathfrak{p}_{1}}$. Since $s_{1}^{*}$ is a homomorphism, $2^{i+1} z=x-y$. Set $\left(\beta_{i}\right)_{\mathfrak{p}_{1}}=2 z+\frac{1}{2^{i}} y$ and $\left(\beta_{i}\right)_{\mathfrak{p}_{2}}=\frac{1}{2^{i}} y$. Then $\left(2^{i} \beta_{i}\right)_{\mathfrak{p}_{1}}=2^{i+1} z+y=x-y+y=x$ and $\left(2^{i} \beta_{i}\right)_{\mathfrak{p}_{2}}=y$. Now by (2.1)

$$
\left((1-\sigma) \beta_{i}\right)_{\mathfrak{p}_{1}}=2 z+\frac{1}{2^{i}} y-\frac{1}{2^{i}} y=2 z=\left(s_{1}^{*} \chi_{i}\right)_{\mathfrak{p}_{1}} .
$$

Moreover, using (2.1) and, by (2.8), that $\sigma\left(s_{1}^{*}(\chi)\right)=-s_{1}^{*}(\chi)$ for $\chi \in \mathrm{X}^{G}$, we obtain

$$
\left((1-\sigma) \beta_{i}\right)_{\mathfrak{p}_{2}}=-2 z=\left(s_{1}^{*} \chi_{i}\right)_{\mathfrak{p}_{2}} .
$$

For all pairs $\mathfrak{p}_{j}, j=1,2$ considered, then, we have $\left(2^{i} \beta_{i}\right)_{\mathfrak{p}_{j}}=\beta_{\mathfrak{p}_{j}}$ and $\left((1-\sigma) \beta_{i}\right)_{\mathfrak{p}_{j}}=$ $\left(s_{1}^{*} \chi_{i}\right)_{\mathfrak{p}_{j}}$.

Now consider archimedean, inert, or ramified places $\mathfrak{p}$. By 2.1), for any $\gamma \in$ $\operatorname{Br}(l),((1-\sigma) \gamma)_{\mathfrak{p}}=0$ at each such $\mathfrak{p}$. Moreover, by $(2.8)$, for any $\delta \in \mathrm{X}^{G}$, $\sigma\left(s_{1}^{*}(\delta)\right)=-s_{1}^{*}(\delta)$. Hence $\left(s_{1}^{*}(\delta)\right)_{\mathfrak{p}} \in\{0,1 / 2\}$. If $\delta$ is divisible by 2 in $\mathrm{X}^{G}$, then since $s_{1}^{*}$ is a homomorphism we have that $\left(s_{1}^{*}(\delta)\right)_{\mathfrak{p}}=0$ at any such $\mathfrak{p}$. Hence the condition $\left((1-\sigma) \beta_{i}\right)_{\mathfrak{p}}=\left(s_{1}^{*} \chi_{i}\right)_{\mathfrak{p}}$ is always satisfied for any choice of $\left(\beta_{i}\right)_{\mathfrak{p}}$. We choose $\left(\beta_{i}\right)_{\mathfrak{p}}$ for these $\mathfrak{p}$ as follows.

Since $\beta \in D(\operatorname{Br}(l)), \beta_{\mathfrak{p}}=0$ at any archimedean $\mathfrak{p}$. We define $\left(\beta_{i}\right)_{\mathfrak{p}}=0$ at all archimedean $\mathfrak{p}$. Now consider the other $\mathfrak{p}$, which are inert or ramified over $k$. Let $\mathfrak{q}$ denote some such place with $\beta_{\mathfrak{q}}=0$. Now for all such $\mathfrak{p} \neq \mathfrak{q}$, define $\left(\beta_{i}\right)_{\mathfrak{p}}=\frac{1}{2^{i}} \beta_{\mathfrak{p}}$ at such $\mathfrak{p}$. Then for all these $\mathfrak{p}$ considered, we have $\left(2^{i} \beta_{i}\right)_{\mathfrak{p}}=\beta_{\mathfrak{p}}$ and $\left((1-\sigma) \beta_{i}\right)_{\mathfrak{p}}=\left(s_{1}^{*} \chi_{i}\right)_{\mathfrak{p}}$. Now $\sum_{\mathfrak{p}} \beta_{\mathfrak{p}}=\sum_{\mathfrak{p} \neq \mathfrak{q}} \beta_{\mathfrak{p}}=0$ since $\beta_{\mathfrak{q}}=0$. Moreover, $2^{i}\left(\beta_{i}\right)_{\mathfrak{p}}=\beta_{\mathfrak{p}}$ for all $\mathfrak{p} \neq \mathfrak{q}$. Hence $2^{i}\left(\sum_{\mathfrak{p} \neq \mathfrak{q}}\left(\beta_{i}\right)_{\mathfrak{p}}\right)=0$. Set $\left(\beta_{i}\right)_{\mathfrak{q}}=-\sum_{\mathfrak{p} \neq \mathfrak{q}}\left(\beta_{i}\right)_{\mathfrak{p}}$. 
Then $2^{i}\left(\beta_{i}\right)_{\mathfrak{q}}=0=\beta_{\mathfrak{q}}$ and $\sum_{\mathfrak{p}}\left(\beta_{i}\right)_{\mathfrak{p}}=0$. Hence $\left\{\beta_{\mathfrak{p}}\right\}$ defines an element of $\operatorname{Br}(l)_{2}$ satisfying $2^{i} \beta_{i}=\beta$ and $(1-\sigma) \beta_{i}=s_{1}^{*} \chi_{i}$ and we are done.

Lemma 3.2. Suppose $k$ is a number field, and let $\alpha=\beta+\chi \in \mathrm{B}^{G}$. Suppose that $\operatorname{ht}_{\mathrm{B}^{G}}(\alpha)>0$. Then $\mathrm{ht}_{\mathrm{X}^{G}}(\chi)-1 \leq \mathrm{ht}_{\mathrm{B}^{G}}(\alpha) \leq \mathrm{ht}_{\mathrm{X}^{G}}(\chi)$.

Proof. By projection from $\mathrm{B}^{G}$ to $\mathrm{X}^{G}$ we see that $\mathrm{ht}_{\mathrm{B}^{G}}(\alpha) \leq \mathrm{ht}_{\mathrm{X}^{G}}(\chi)$.

Suppose that $h=\mathrm{ht}_{\mathrm{X}^{G}}(\chi)$ lies in $\mathbb{N}$. Since $\operatorname{ht}_{\mathrm{B}^{G}}(\alpha)>0, \beta_{\mathfrak{p}}=0$ at all archimedean $\mathfrak{p}$ and $\beta \in D(\operatorname{Br}(l))$. By Lemma 3.1 $\mathrm{ht}_{\mathrm{B}^{G}}(\alpha) \geq \mathrm{ht}_{\mathrm{X}^{G}}(\chi)-1$. Now suppose that $h=\omega$. In this case Lemma 3.1] shows that in fact ht $\mathrm{B}^{G}(\alpha)=\mathrm{ht}_{\mathrm{X}^{G}}(\chi)$. Since the Ulm length of $\mathrm{X}^{G}$ is less than or equal to $\omega 2$ [FS, Thm. 1], we are left with the cases $h=\omega+n$ for some $n, h=\omega 2$, and $h=\infty$.

For each $i \geq 1$ such that $h \geq \omega+i$, we do the following. Let $\chi_{i} \in \mathrm{X}^{G}$ be such that $2^{i} \chi_{i}=\chi$ and for each $j \in \mathbb{N}$ let $\delta_{j} \in \mathrm{X}^{G}$ satisfy $2^{j} \delta_{j}=\chi_{i}$. The proof of Lemma 3.1 shows that we may find $\beta_{i-1}$ such that $\alpha_{i-1}=\beta_{i-1}+\chi_{i-1} \in \mathrm{B}^{G}$ and $2^{i-1} \alpha_{i-1}=\alpha$. By construction $\left(\beta_{i-1}\right)_{\mathfrak{p}}=0$ at all archimedean $\mathfrak{p}$, and so $\beta_{i-1} \in D(\operatorname{Br}(l))$. Now $\operatorname{ht}_{X^{G}}\left(\chi_{i-1}\right) \geq \omega$, and hence we may apply Lemma 3.1 again to show that ht $\mathrm{B}^{G}\left(\alpha_{i-1}\right) \geq \omega$. Hence ht $\mathrm{B}^{G}(\alpha) \geq \omega+(i-1)$. If $h=\omega+n$ for some $n$, we have that $\omega+(n-1)=\operatorname{ht}_{X^{G}}(\chi)-1 \leq \operatorname{ht}_{B^{G}}(\alpha)$. If $h=\omega 2$, we have shown that $\operatorname{ht}_{\mathrm{B}^{G}}(\alpha)=\omega 2$. A similar argument handles the case $h=\infty$, where $\operatorname{ht}_{\mathrm{B}^{G}}(\alpha)=\infty$ as well.

Lemma 3.3. Suppose that $k$ is a number field, $\chi \in \mathrm{X}^{G}$, and $\mathrm{ht}_{\mathrm{X}^{G}}(\chi)>1$. Then there exists $\alpha=\beta+\chi \in \mathrm{B}^{G}$ such that $|\alpha|=|\chi|$ and $\mathrm{ht}_{\mathrm{X}^{G}}(\chi)-1 \leq \mathrm{ht}_{\mathrm{B}^{G}}(\alpha) \leq$ $\mathrm{ht}_{\mathrm{X}^{G}}(\chi)$.

Proof. Since $\mathrm{ht}_{\mathrm{X}^{G}}(\chi)>1, \chi=4 \epsilon$ for some $\epsilon \in \mathrm{X}^{G}$. Let $\delta=2 \epsilon$ and $\gamma^{\prime}=s_{1}^{*}(\delta)$. Since $h_{X^{G}}(\delta)>0, \gamma_{\mathfrak{p}}^{\prime}=0$ at each archimedean place $\mathfrak{p}$ of $l$. Similarly, since $\mathrm{ht}_{\mathrm{X}^{G}}(\delta)>0$ and since by (2.8) the image $s_{1}^{*}\left(\mathrm{X}^{G}\right)$ is $\sigma$-negated, we have that $\gamma_{\mathfrak{p}}^{\prime}=0$ at every inert or ramified place $\mathfrak{p}$ of $l$, and, by (2.1), that at every pair $\mathfrak{p}_{1}, \mathfrak{p}_{2}$ of $\sigma$-permuted places of $l, \gamma_{\mathfrak{p}_{1}}^{\prime}=-\gamma_{\mathfrak{p}_{2}}^{\prime}$ as well.

We will define a $\gamma \in \operatorname{Br}(l)_{2}$ via its invariants. Let $\gamma_{\mathfrak{p}}=0$ at every archimedean place of $l$. For every pair $\mathfrak{p}_{1}, \mathfrak{p}_{2}$ of $\sigma$-permuted places of $l$, let $\gamma_{\mathfrak{p}_{1}}=\gamma_{\mathfrak{p}_{1}}^{\prime}$ and $\gamma_{\mathfrak{p}_{2}}=0$. Now there are only finitely many such pairs $\mathfrak{p}_{1}, \mathfrak{p}_{2}$ at which $\gamma^{\prime}$ has nontrivial invariants. For each such pair $\mathfrak{p}_{1}, \mathfrak{p}_{2}$ choose an inert or ramified place $\mathfrak{q}$ of $l$ and set $\gamma_{\mathfrak{q}}=-\gamma_{\mathfrak{p}_{1}}$. At all other inert or ramified places $\mathfrak{q}$ set $\gamma_{\mathfrak{q}}=0$.

By construction $\sum_{\mathfrak{p}} \gamma_{\mathfrak{p}}=0$, so there exists a $\gamma \in \operatorname{Br}(l)_{2}$ with invariants $\left\{\gamma_{\mathfrak{p}}\right\}$. Furthermore, $((1-\sigma) \gamma)_{\mathfrak{p}}=0$ at all inert, ramified, or archimedean places $\mathfrak{p}$ by (2.1). At pairs $\mathfrak{p}_{1}, \mathfrak{p}_{2}$ of $\sigma$-permuted places, we have

$$
((1-\sigma) \gamma)_{\mathfrak{p}_{1}}=\gamma_{\mathfrak{p}_{1}}^{\prime}=-\gamma_{\mathfrak{p}_{2}}^{\prime}=((1-\sigma) \gamma)_{\mathfrak{p}_{2}}
$$

Hence $(1-\sigma) \gamma=2(1-\sigma) \gamma^{\prime}=2 s_{1}^{*}(\delta)=s_{1}^{*}(2 \delta)=s_{1}^{*} \chi$ and $\gamma+\delta \in \mathrm{B}^{G}$. Since the invariants of $\gamma$ are either 0 or equal to a corresponding invariant of $\gamma^{\prime}$, which is a homomorphic image of $\delta,|\gamma| \leq|\delta|$, and hence $|\gamma+\delta|=|\delta|$. Setting $\beta=2 \gamma$ and $\alpha=2(\gamma+\delta)=\beta+\chi$, we then have $|\alpha|=|\chi|$. Moreover, $\operatorname{ht}_{\mathrm{B}^{G}}(\alpha)>0$ since $\alpha=2(\gamma+\delta)$. Using Lemma 3.2, we have that ht $\mathrm{X}^{G}(\chi)-1 \leq \operatorname{ht}_{\mathrm{B}^{G}}(\alpha) \leq \mathrm{ht}_{\mathrm{X}^{G}}(\chi)$.

Proof of Proposition [5. $\Rightarrow$ Let $\alpha=\beta+\chi$ be an order 2 element in $\mathrm{B}^{G}$ with $\operatorname{ht}_{\mathrm{B}^{G}}(\alpha)=\omega 2$. By restriction, we have that $\operatorname{ht}_{\mathrm{B}^{G}}(\alpha) \leq \mathrm{ht}_{\mathrm{X}^{G}}(\chi)$. Since the Ulm length of $\mathrm{X}$ is at most $\omega 2\left[\mathrm{FS}\right.$, Thm. 1], we have that $\operatorname{ht}_{\mathrm{X}^{G}}(\chi) \in\{\omega 2, \infty\}$. 
Suppose that $\chi \in D\left(\mathrm{X}^{G}\right)$. By Lemma 3.3 there exists $\beta^{\prime} \in \operatorname{Br}(l)_{2}$ such that $\alpha^{\prime}=\beta^{\prime}+\chi \in \mathrm{B}^{G},\left|\alpha^{\prime}\right|=|\chi|$, and $\alpha^{\prime} \in D\left(\mathrm{~B}^{G}\right)$. But then $\gamma=\alpha-\alpha^{\prime}=\beta-\beta^{\prime} \in \mathrm{B}^{G}$ satisfies $\operatorname{ht}_{\mathrm{B}^{G}}(\gamma)=\omega 2$. Now an element $b \in \operatorname{Br}(l)_{2}$ lies in $\mathrm{B}^{G}$ if and only if $(1-\sigma) b=0$. But $\operatorname{Br}(l)_{2}^{\langle\sigma\rangle}$ consists, by [2.1), of a restricted direct sum of $\mathbb{Z} / 2 \mathbb{Z}$ and $\mathbb{Q}_{2} / \mathbb{Z}_{2}$ summands. Therefore there is no element in $\operatorname{Br}(l)_{2} \cap \mathrm{B}^{G}$ of height $\omega 2$ and we have a contradiction. Therefore $\mathrm{ht}_{\mathrm{X}^{G}}(\chi)=\omega 2$.

$(\Leftarrow)$ Now suppose that $\chi \in \mathrm{X}^{G}$ is an order 2 element of height $\omega 2$. By Lemma 3.3 there exists a $\beta \in \operatorname{Br}(l)$ such that $\alpha=\beta+\chi \in \mathrm{B}^{G}$ is of order 2 and $\operatorname{ht}_{\mathrm{B}^{G}}(\alpha)=\omega 2$, and we are done.

\subsection{Reducing to $\lambda$.}

Proposition 6. Suppose $k$ is a number field. Then there exists an order 2 element in $\mathrm{B}^{G}$ of height $\omega 2$ if and only if $\lambda_{w}=\omega 2$ for some nontrivial $w \in W_{I}$.

Lemma 3.4. Suppose $k$ is a number field and $\sum \chi_{p} \in \mathrm{X}_{\neq u}^{G}$ is an order 2 element with height greater than the Ulm length of $\mathrm{X}(l)_{2}$. Then Cor $\sum \chi_{p} \in W$.

Proof. If Cor $\sum \chi_{p}=0$, then $0 \in W$ and we are done.

Otherwise, let $w=\operatorname{Cor} \sum \chi_{p}$. The order of $w$ is 2, and, since by (2.7), Cor is a homomorphism from $\mathrm{X}_{\neq u}^{G}$ to the $\sigma$-invariant subgroup of $\mathrm{X}(l), w \in D\left(\mathrm{X}(l)_{2}^{\langle\sigma\rangle}\right) \subset$ $D\left(\mathrm{X}(l)_{2}\right)^{\langle\sigma\rangle}$. Hence $w \in I \oplus{ }_{2} N \oplus P^{\langle\sigma\rangle}$.

Consider $p \neq \tilde{p}$ for which Cor $\chi_{p}$ is not trivial. By (2.5),

$$
\operatorname{Cor}\left(\chi_{p}+\chi_{\tilde{p}}\right)=\operatorname{Cor}\left(\chi_{p}+s_{p \tilde{p}}^{*}\left(\chi_{p}\right)\right)=(1+\sigma) \operatorname{Cor} \chi_{p}
$$

Now Cor $\chi_{p}$ is an element of order at most two in $\mathrm{X}(l)_{2}$, therefore represented by $l(\sqrt{e})$ for $e \in l^{*}$. Then $(1+\sigma)$ Cor $\chi_{p}$ is represented by $l\left(\sqrt{N_{l / k}(e)}\right)$. Set $z_{p \tilde{p}}=N_{l / k}(e)$.

Now consider $p=\tilde{p}$ for which Cor $\chi_{p}$ is not trivial. Then as in the proof of (2.6), there exists a $\tau \in G_{l}$ such that $a_{p}=c / \sigma \tau\left(a_{p}\right)$ and we let $\tilde{\sigma}=\sigma \tau$. Then $\tilde{\sigma}$ is an automorphism of $l\left(a_{p}\right)$ of order 2 with fixed field $k_{p}:=k\left(a_{p}+c / a_{p}\right)$.

We claim that $\left[k_{p}: k\right]$ is even. Since $\sqrt{d} \notin k_{p}$ and $\left[l\left(a_{p}\right): k_{p}\right]=2, l\left(a_{p}\right)=k_{p}(\sqrt{d})$. Then $a_{p} \in l\left(a_{p}\right)$ satisfies $N_{l\left(a_{p}\right) / k_{p}} a_{p}=c$, so the quaternion algebra $(c, d)$ splits over $k_{p}$. But then $\left[k_{p}: k\right]$ is even.

Since $\chi_{p} \in \mathrm{X}\left(l\left(a_{p}\right)\right)$ is of order 2, it is represented by $l\left(a_{p}\right)(\sqrt{f})$, where $f$ is determined up to its class in $l\left(a_{p}\right)^{*} / l\left(a_{p}\right)^{* 2}$. Moreover, since $s_{p p}^{*}\left(\chi_{p}\right)=\chi_{p}$, by (2.6) $), \tilde{\sigma}\left(\chi_{p}\right)=\chi_{p}$, or $\tilde{\sigma}(f)=f$ in $l\left(a_{p}\right)^{*} / l\left(a_{p}\right)^{* 2}$. Hence $N_{l\left(a_{p}\right) / k_{p}}(f) \in l\left(a_{p}\right)^{* 2}$. By Kummer theory, $\left(k_{p}^{*} \cap l\left(a_{p}\right)^{* 2}\right) / k_{p}^{* 2}$ consists only of the classes 1 and $d$. Therefore, modulo $k_{p}^{* 2}, N_{l\left(a_{p}\right) / k_{p}}(f)$ is either 1 or $d$.

Now Cor $\chi_{p}$ is represented by $l(\sqrt{e})$, where $e=N_{l\left(a_{p}\right) / l}(f)$, and then $N_{l / k}(e)=$ $N_{k_{p} / k} N_{l\left(a_{p}\right) / k_{p}}(f)$. Since $N_{l\left(a_{p}\right) / k_{p}}(f)$ is 1 or $d \bmod k_{p}^{* 2}$ and

$$
N_{k_{p} / k}\left(d z^{2}\right)=d^{\left[k_{p}: k\right]} N_{k_{p} / k}(z)^{2} \in k^{* 2}
$$

we deduce that $N_{l / k}(e)$ is a square in $k^{*}$. Then, from the square-class exact sequence ([La, Thm. 3.4])

$$
1 \rightarrow\left\langle d \cdot k^{* 2}\right\rangle \rightarrow k^{*} / k^{* 2} \rightarrow l^{*} / l^{* 2} \stackrel{N_{l / k}}{\longrightarrow} k^{*} / k^{* 2}
$$

we have that, up to squares in $l^{*}, e$ is represented by an element of $k^{*}$. Set $z_{p p}$ to be this value.

For all remaining $p$, set $z_{p \tilde{p}}=1$. 
Now Cor $\sum \chi_{p}$ is represented by $l(\sqrt{e})$, where $e$ is the product in $l^{*}$ of $z_{p \tilde{p}}$ for all $\{p, \tilde{p}\}$. Hence Cor $\sum \chi_{p}$ is an element of order at most 2 represented by $l(\sqrt{e})$, where $e$ is a product of elements from $k$.

Lemma 3.5. Suppose $k$ is a number field and $\chi \in \mathrm{X}^{G}$ is an order 2 element with $\operatorname{ht}_{\mathrm{X}^{G}}(\chi)=\omega 2$. Then there exists $\hat{\chi} \in D\left(\mathrm{X}^{G}\right)$ with $|\hat{\chi}|=2$ such that $w=\chi-\hat{\chi} \in W_{I}$ is nontrivial and $\lambda_{w}=\omega 2$.

Proof. Write $\chi=\chi_{u}+\chi_{\neq u}$ with $\chi_{\neq u}=\sum \chi_{p}$. Since for each $p$ the Ulm length of $\mathrm{X}\left(l\left(a_{p}\right)\right)_{2}$ is $\omega+n$ for some $n \in \mathbb{N}_{\geq 0}$ [FS, Thm. 1] and since $\mathrm{ht}_{\mathrm{X}^{G}}(\chi)=\omega 2$, we have $\chi_{\neq u} \in D\left(\mathrm{X}_{\neq u}\right)$. For each summand $\mathrm{X}\left(l\left(a_{p}\right)\right)_{2}$ with $p=\tilde{p}$, and for each pair of summands $\mathrm{X}\left(l\left(a_{p}\right)\right)_{2} \oplus \mathrm{X}\left(l\left(a_{\tilde{p}}\right)\right)_{2}$ for $p \neq \tilde{p}$, the divisible subgroup is a finite direct sum of $\mathbb{Q}_{2} / \mathbb{Z}_{2}$ components [NSW Thm. 11.1.2].

The fixed subgroup of an order 2 action on a finite direct sum of $\mathbb{Q}_{2} / \mathbb{Z}_{2}$ components is a direct sum of $\mathbb{Z} / 2 \mathbb{Z}$ and $\mathbb{Q}_{2} / \mathbb{Z}_{2}$ components. Hence for $\tilde{p}=p$ the $s_{p p}^{*}$-fixed subgroup of $D\left(\mathrm{X}\left(l\left(a_{p}\right)\right)_{2}\right)$, and when $\tilde{p} \neq p$, the $\left(s_{p \tilde{p}}+s_{\tilde{p} p}\right)^{*}$-fixed subgroup of $D\left(\mathrm{X}\left(l\left(a_{p}\right)\right)_{2} \oplus \mathrm{X}\left(l\left(a_{\tilde{p}}\right)\right)_{2}\right)$, is a direct sum of $\mathbb{Z} / 2 \mathbb{Z}$ and $\mathbb{Q}_{2} / \mathbb{Z}_{2}$ components. Since $\chi_{\neq u} \in 2 \mathrm{X}_{\neq u}^{G}$, in each summand or pair of summands, then, the components of $\chi_{\neq u}$ lie in the divisible part of the $\sum_{p} s_{p \tilde{p}}^{*}$-fixed subgroup. Hence $\chi_{\neq u} \in D\left(\mathrm{X}_{\neq u}^{G}\right)$. For each $p$, let $\left\{\chi_{p}^{(i)}\right\}_{i=0}^{\infty}$ be a divisible tower over $\chi_{p}$, so that $\left\{\sum \chi_{p}^{(i)}\right\}_{i=0}^{\infty} \subset X_{\neq u}^{G}$ is a divisible tower over $\chi \neq u$.

Since $\mathrm{ht}_{\mathrm{X}^{G}}(\chi)=\omega 2$ and the Ulm length of $\mathrm{X}(l)_{2}$ is $\omega+n$ for some $n \in \mathbb{N}_{\geq 0}$, $\chi_{u} \in D\left(\mathrm{X}(l)_{2}\right)$. Following the decomposition of $D\left(\mathrm{X}(l)_{2}\right)$ in (3.1), write $\chi_{u}=$ $w_{I}+w_{N}+w_{P}$.

For each pair $\mathbb{Q}_{2} / \mathbb{Z}_{2} \oplus \mathbb{Q}_{2} / \mathbb{Z}_{2}$ of $\sigma$-permuted summands in $P$, denote the components of $z \in P$ in these summands by $z_{s}$ and $z_{t}$. Define

$$
\left(w_{P}^{(i)}\right)_{s}=-\left(\operatorname{Cor} \sum \chi_{p}^{(i)}\right)_{s}-\frac{1}{2^{i}}\left(w_{P}\right)_{t}
$$

and $\left(w_{P}^{(i)}\right)_{t}=\frac{1}{2^{i}}\left(w_{P}\right)_{t}$. (We denote by $\left(1 / 2^{i}\right)\left(w_{P}\right)_{t}$ some element yielding $\left(w_{P}\right)_{t}$ under multiplication by $2^{i}$, and we fix this element for the duration.) Then $\left|w_{P}^{(i)}\right| \leq$ $2^{i+1}$ and $\left\{w_{P}^{(i)}\right\}_{i=0}^{\infty}$ is a divisible tower over $w_{P}^{(0)}=w_{P}$ since the fact that $(1+\sigma) w_{P}=$ $\left(-\operatorname{Cor} \sum \chi_{p}\right)_{P}$ implies $\left((1+\sigma) w_{P}\right)_{s}=\left(-\operatorname{Cor} \sum \chi_{p}\right)_{s}$ and $\left(w_{P}\right)_{s}=\left(-\operatorname{Cor} \sum \chi_{p}\right)_{s}-$ $\left(w_{P}\right)_{t}$.

Now

$$
\left((1+\sigma) w_{P}^{(i)}\right)_{s}=-\left(\operatorname{Cor} \sum \chi_{p}^{(i)}\right)_{s}=\left((1+\sigma) w_{P}^{(i)}\right)_{t} .
$$

Furthermore, by (2.7) the image of Cor on the divisible tower $\left\{\sum \chi_{p}^{(i)}\right\}_{i=0}^{\infty}$ over $\chi_{\neq u}=\sum \chi_{p}$ lies in the $\sigma$-invariant part of $P$, hence with components lying in the diagonals of the pairs $\mathbb{Q}_{2} / \mathbb{Z}_{2} \oplus \mathbb{Q}_{2} / \mathbb{Z}_{2}$. Hence $\left((1+\sigma) w_{P}^{(i)}\right)_{t}=-\left(\operatorname{Cor} \sum \chi_{p}^{(i)}\right)_{t}$ as well.

Since $N$ is divisible, we may choose a divisible tower $\left\{w_{N}^{(i)}\right\}_{i=0}^{\infty} \subset N$ over $w_{N}$. Now $(1+\sigma) w_{N}^{(i)}=0$ since $\sigma$ acts by negation on $N$. Furthermore, by (2.7) the image of Cor on the divisible tower over $\chi_{\neq u}$ lies in $D\left(\mathrm{X}(l)_{2}\right)$ and is $\sigma$-invariant, hence has zero component in $N$. Hence $(1+\sigma)\left(w_{N}^{(i)}+w_{P}^{(i)}\right)=-\left(\operatorname{Cor} \sum \chi_{p}^{(i)}\right)_{N \oplus P}$.

Finally set $\hat{w}_{I}^{(i)}=\left(-\operatorname{Cor} \sum \chi_{p}^{(i+1)}\right)_{I}$. Then since $\sigma$ is invariant on $I$,

$$
(1+\sigma)\left(\hat{w}_{I}^{(i)}\right)=2 \hat{w}_{I}^{(i)}=2\left(-\operatorname{Cor} \sum \chi_{p}^{(i+1)}\right)_{I}=\left(-\operatorname{Cor} \sum \chi_{p}^{(i)}\right)_{I} .
$$


Moreover, $\left\{\hat{w}_{I}^{(i)}\right\}_{i=0}^{\infty}$ is a divisible tower over $\hat{w}_{I}:=\hat{w}_{I}^{(0)}$. Note that $\hat{w}_{I}$ has order at most 2 because $\left(-\operatorname{Cor} \sum \chi_{p}\right)_{I}=\left((1+\sigma) \chi_{u}\right)_{I}=(1+\sigma) w_{I}=0$ since $\chi \in \mathrm{X}^{G}$ and $w_{I}$ is of order 2 .

Let $\hat{\chi}_{u}^{(i)}=\hat{w}_{I}^{(i)}+w_{N}^{(i)}+w_{P}^{(i)}$ and $\hat{\chi}^{(i)}=\hat{\chi}_{u}^{(i)}+\sum \chi_{p}^{(i)}$. Then $\left\{\hat{\chi}^{(i)}\right\}_{i=0}^{\infty} \subset \mathrm{X}^{G}$ is a divisible tower over $\hat{\chi}:=\hat{\chi}_{u}^{(0)}+\sum \chi_{p}$. Clearly $w:=\chi-\hat{\chi}=w_{I}-\hat{w}_{I} \in I$ and $|w| \leq 2$. If $w=0$, then $\chi=\hat{\chi}$ and we have a contradiction: $\chi \in D\left(\mathrm{X}^{G}\right)$. Hence $|w|=2$ and $\operatorname{ht}_{X^{G}}(w)=w 2$.

Now since ht $\mathrm{X}^{G}(w)=\omega 2$, for any $n \in \mathbb{N}$ there exists a $\chi^{\prime} \in \mathrm{X}^{G}$ of height $\omega+n$ and $w=2 \chi^{\prime}$. We restrict $\omega+n$ to ordinals greater than the Ulm length of $\mathrm{X}(l)_{2}$. Write $\chi^{\prime}=\chi_{u}^{\prime}+\sum \chi_{p}^{\prime}$. Then $\chi_{u}^{\prime} \in D\left(\mathrm{X}(l)_{2}\right)$. Moreover, $(1+\sigma) \chi_{u}^{\prime}=-\operatorname{Cor} \sum \chi_{p}^{\prime}$. Now $\left|\sum \chi_{p}^{\prime}\right|$ is at most 2 since $w=2 \chi^{\prime}$ has no component in $X_{\neq u}$. If the order is 2 , then by Lemma 3.4 - Cor $\sum \chi_{p}^{\prime}$ lies in $W$; if the order is 1 , then - Cor $\sum \chi_{p}^{\prime}=0 \in W$.

Since $\chi_{u}^{\prime} \in D\left(\mathrm{X}(l)_{2}\right)$, write $\chi_{u}^{\prime}=\chi_{I}^{\prime}+\chi_{N}^{\prime}+\chi_{P}^{\prime}$ according to (3.1). Since $2 \chi^{\prime}=w \in I, \chi_{N}^{\prime}$ and $\chi_{P}^{\prime}$ are of order at most 2 . Hence $(1+\sigma) \chi_{I}^{\prime}=2 \chi_{I}^{\prime}=w_{I}=w$ and $(1+\sigma) \chi_{N}^{\prime}=2 \chi_{N}^{\prime}=0$. Therefore $(1+\sigma) \chi_{u}^{\prime}=-\operatorname{Cor} \sum \chi_{p}^{\prime}=w+(1+\sigma) \chi_{P}^{\prime} \in W$. Therefore $\left(-\operatorname{Cor} \sum \chi_{p}^{\prime}\right)_{I}=w$, and $\operatorname{ht}_{\mathrm{X}_{\neq u}^{G}}\left(\sum \chi_{p}^{\prime}\right) \geq \mathrm{ht}_{\mathrm{X}^{G}}\left(\chi^{\prime}\right)=\omega+n$. Hence, by considering $-\sum \chi_{p}^{\prime}$ instead of $\sum \chi_{p}^{\prime}$, we have $\lambda_{w} \geq \omega 2$.

Now suppose that $\lambda_{w}=\infty$. Then since the Ulm length of $X_{\neq u}^{G}$ is at most $\omega 2[\mathrm{FS}$ ] Thm. 1], there exists $\chi_{\neq u}=\sum \chi_{p} \in \mathrm{X}_{\neq u}^{G}$ of order at most 2 with $\mathrm{ht}_{\mathrm{X}_{\neq u}^{G}}\left(\chi_{\neq u}\right)=\infty$ and $\left(\text { Cor } \chi_{\neq u}\right)_{I}=w$. Let $\left\{\sum \chi_{p}^{(i)}\right\}_{i=0}^{\infty} \subset \mathrm{X}_{\neq u}^{G}$ be a divisible tower over $\chi_{\neq u}$. Now let $\hat{P}$ be a finite direct sum of $\mathbb{Q}_{2} / \mathbb{Z}_{2}$ summands in $P$ such that $\hat{P} \oplus \sigma(\hat{P})=P$. For each $i \geq 1$, set $\chi_{u}^{(i)}=\left(-\operatorname{Cor} \sum \chi_{p}^{(i+1)}\right)_{I}+\left(-\operatorname{Cor} \sum \chi_{p}^{(i)}\right)_{\hat{P}}$. Then

$$
\left((1+\sigma)\left(\chi_{u}^{(i)}\right)\right)_{I}=2\left(\chi_{u}^{(i)}\right)_{I}=\left(-2 \operatorname{Cor} \sum \chi_{p}^{(i+1)}\right)_{I}=\left(-\operatorname{Cor} \sum \chi_{p}^{(i)}\right)_{I}
$$

and

$$
\left((1+\sigma)\left(\chi_{u}^{(i)}\right)\right)_{\hat{P}}=\left(\chi_{u}^{(i)}\right)_{\hat{P}}=\left(-\operatorname{Cor} \sum \chi_{p}^{(i)}\right)_{\hat{P}} .
$$

Moreover, since by (2.7) the image of Cor is $\sigma$-invariant on $\mathrm{X}_{\neq u}^{G}$, the equality holds over $\sigma(\hat{P})$ as well, and holds over $N$ since both sides must be trivial on $N$. Then for $i \geq 0, \chi_{u}^{(i)}+\sum \chi_{p}^{(i)} \in \mathrm{X}^{G}$. Now

$$
\begin{aligned}
2 \chi_{u}^{(0)} & =2\left(\left(-\operatorname{Cor} \sum \chi_{p}^{(1)}\right)_{I}+\left(-\operatorname{Cor} \sum \chi_{p}^{(0)}\right)_{\hat{P}}\right) \\
& =\left(-\operatorname{Cor} \sum \chi_{p}^{(0)}\right)_{I}=-w .
\end{aligned}
$$

Since $|w| \leq 2,-w=w$. Let $\chi_{0}^{\prime}=w$ and for $i \geq 1, \chi_{i}^{\prime}=\chi_{u}^{(i-1)}+\sum \chi_{p}^{(i-1)}$. Then $2 \chi_{1}^{\prime}=w$ and $\left\{\chi_{i}^{\prime}\right\}_{i=0}^{\infty} \subset \mathrm{X}^{G}$ is a divisible tower over $w$, so $w \in D\left(\mathrm{X}^{G}\right)$. But then $\chi=w+\hat{\chi} \in D\left(\mathrm{X}^{G}\right)$, a contradiction.

Proof of Proposition [6. $(\Rightarrow)$ By Proposition [5, if there is an order 2 element in $\mathrm{B}^{G}$ of height $\omega 2$, then there is an order 2 element in $\mathrm{X}^{G}$ of height $\omega 2$. By Lemma 3.5 there exists a nontrivial $w \in W_{I}$ with $\lambda_{w}=\omega 2$.

$(\Leftarrow)$ Suppose that $\lambda_{w}=\omega 2$ for some nontrivial $w \in W_{I}$. We claim that there is an order 2 element $\hat{w}$ in $\mathrm{X}^{G}$ with $\mathrm{ht}_{\mathrm{X}^{G}}(\hat{w})=\omega 2$.

Since $\lambda_{w}=\omega 2$, for each ordinal $\omega+i$ greater than the Ulm length of $\mathrm{X}(l)_{2}$ there exists an element $\chi_{\neq u}=\sum \chi_{p} \in \mathrm{X}_{\neq u}^{G}$ with ht $\mathrm{X}_{\neq u}^{G}\left(\chi_{\neq u}\right)=\omega+i$ and $-\left(\operatorname{Cor} \chi_{\neq u}\right)_{I}=$ $-w=w$. 
For each such ordinal $\omega+i$, we proceed as follows. Let $\chi_{\neq u}^{(i)} \in \mathrm{X}_{\neq u}^{G}$ be an element such that $2^{i} \chi_{\neq u}^{(i)}=\chi_{\neq u}$ and for each $j \in \mathbb{N}$ let $\delta_{\neq u}^{(j)} \in \mathrm{X}_{\neq u}^{G}$ satisfy $2^{j} \delta_{\neq u}^{(j)}=\chi_{\neq u}^{(i)}$. Now set $\chi_{u}^{(i)}=-\operatorname{Cor} \chi_{\neq u}^{(i)}$ and $\chi^{(i)}=\chi_{u}^{(i)}+\chi_{\neq u}^{(i-1)}$. Then since by (2.7) the image of - Cor on $\mathrm{X}_{\neq u}^{G}$ is $\sigma$-invariant,

$$
(1+\sigma) \chi_{u}^{(i)}=2 \chi_{u}^{(i)}=-2 \operatorname{Cor} \chi_{\neq u}^{(i)}=-\operatorname{Cor} \chi_{\neq u}^{(i-1)},
$$

and we have $\chi^{(i)} \in \mathrm{X}^{G}$. Moreover, $2^{i} \chi^{(i)}=-\operatorname{Cor} \chi_{\neq u}$ with $-\left(\operatorname{Cor} \chi_{\neq u}\right)_{I}=w$. Continuing on, for each $j \geq 2$, set $\delta_{u}^{(j)}=-\operatorname{Cor} \delta_{\neq u}^{(j)}$ and $\delta^{(j)}=\delta_{u}^{(j)}+2 \delta_{\neq u}^{(j)}$; for $j=1$ set $\delta_{u}^{(1)}=-\operatorname{Cor} \delta_{\neq u}^{(1)}$ and $\delta^{(1)}=\delta_{u}^{(1)}+\chi_{\neq u}^{(i)}$. Then $2^{j} \delta^{(j)}=\chi^{(i)}$ and as before, $\delta^{(j)} \in \mathrm{X}^{G}$ for each $j$. Hence we have shown that $w^{\prime}=-$ Cor $\chi_{\neq u}$ is an order 2 element in $\mathrm{X}(l)^{\langle\sigma\rangle}$ with $\operatorname{ht}_{\mathrm{X}^{G}}\left(w^{\prime}\right) \geq \omega+i$ and $w_{I}^{\prime}=w$. In fact, because the height is greater than the Ulm length of $\mathrm{X}(l)_{2}, w^{\prime} \in D\left(\mathrm{X}(l)_{2}\right)^{\langle\sigma\rangle}$.

Hence for every $\omega+i$ we have produced an order 2 element $w_{i}^{\prime} \in D\left(\mathrm{X}(l)_{2}\right)^{\langle\sigma\rangle}$ which satisfies $\operatorname{ht}_{\mathrm{X}^{G}}\left(w_{i}^{\prime}\right) \geq \omega+i$ and $\left(w_{i}^{\prime}\right)_{I}=w$. Now the divisible subgroup of $\mathrm{X}(l)_{2}$ is a finite direct sum of $\mathbb{Q}_{2} / \mathbb{Z}_{2}$ summands [NSW] Thm. 11.1.2], and hence its exponent 2 subgroup is finite. Hence for some $\hat{w} \in D\left(\mathrm{X}(l)_{2}\right)^{\langle\sigma\rangle}, \mathrm{ht}_{\mathrm{X}}{ }(\hat{w}) \geq \omega+i_{n}$ for an unbounded strictly increasing sequence $\left\{i_{n}\right\}_{n=1}^{\infty}$ of natural numbers. Therefore there exists a $\hat{w}$ of order 2 in $D(\mathrm{X}(l))^{\langle\sigma\rangle} \subset \mathrm{X}^{G}$ with $\mathrm{ht}_{\mathrm{X}^{G}}(\hat{w}) \geq \omega 2$ and $\hat{w}_{I}=w$.

Now suppose that $\hat{w}$ is divisible in $\mathrm{X}^{G}$. Let $\left\{\chi^{(i)}\right\}_{i=0}^{\infty} \subset \mathrm{X}^{G}$ be a divisible tower over $\hat{w}$. Write $\chi^{(i)}=\chi_{u}^{(i)}+\chi_{\neq u}^{(i)}$ for each $i$, and consider $\chi^{\prime}=\chi_{\neq u}^{(1)}$, necessarily of order less than or equal to 2 since $\chi_{\neq u}^{(0)}=0$ because $\hat{w} \in \mathrm{X}(l)_{2}$. Then $\left\{\chi_{\neq u}^{(i+1)}\right\}_{i=0}^{\infty} \subset$ $\mathrm{X}_{\neq u}^{G}$ is a divisible tower over $\chi^{\prime}$. Since $\chi^{(1)} \in \mathrm{X}^{G}$, we have that $(1+\sigma) \chi_{u}^{(1)}=$ $-\operatorname{Cor} \chi_{\neq u}^{(1)}$. Restricting the equation to $I$, we have $\left(\chi_{u}^{(0)}\right)_{I}=\left(-\operatorname{Cor} \chi^{\prime}\right)_{I}$, and the left-hand side is in fact $(\hat{w})_{I}=w$. Hence $\chi^{\prime}$ is an order 2 element in $\mathrm{X}_{\neq u}^{G}$ with $\left(\operatorname{Cor} \chi^{\prime}\right)_{I}=w$; we then have that $\lambda_{w}=\infty$, a contradiction.

\section{Proof of the Main Theorem}

Proof. We show that any order two element $\alpha$ in $\operatorname{Br}(E)$ of height at least $\omega 2$ is divisible.

Suppose $\alpha$ is an order two element in $\operatorname{Br}(E)=H^{2}\left(G_{k}, \bar{k}(u)^{*}\right)$ of height at least $\omega 2$. Then the image $\phi(\alpha)$ of $\alpha$ in (1.1) is an element of $\mathrm{B}^{G}$ of height at least $\omega 2$.

Since the Leopoldt conjecture holds for $l$ and 2 , there is only one $\mathbb{Z}_{2}$-extension of $l$. Since $l$ is the quadratic subextension of $k^{c y c} / k$, the $\mathbb{Z}_{2}$-extension of $l$ is precisely $k^{c y c}$. Hence $D\left(\mathrm{X}(l)_{2}\right) \cong \mathbb{Q}_{2} / \mathbb{Z}_{2}$ and $\sigma$ acts trivially on $D\left(\mathrm{X}(l)_{2}\right)$. In the decomposition of $D\left(\mathrm{X}(l)_{2}\right)$ in (B.1), $N=P=0$ and $I \cong \mathbb{Q}_{2} / \mathbb{Z}_{2}$. But since the quadratic subextension of $k^{c y c} / l$ is cyclic of order 4 over $k, W=0$. Hence by Proposition 6 there exists no order 2 element of $\mathrm{B}^{G}$ of height $\omega 2$. Hence $\phi(\alpha)$ is divisible.

If $\phi(\alpha) \neq 0$ and $\left\{\tilde{\alpha}_{i}\right\}$ is a divisible tower over $\phi(\alpha)$, then we may find preimages $\alpha_{n} \in \operatorname{Br}(E)$ such that $\phi\left(\alpha_{n}\right)=\tilde{\alpha}_{n}$ and $2^{m} \alpha_{n}=\alpha_{n-m}$, as follows. Let $\alpha_{n}=$ $2 \cdot \phi^{-1}\left(\tilde{\alpha}_{n+1}\right)$. Since the kernel of $\phi$ is the relative Brauer group $\operatorname{Br}(E l / E)$ and is therefore of exponent 2 , this map is well defined. We calculate

$$
\phi\left(\alpha_{n}\right)=\phi\left(2 \cdot \phi^{-1}\left(\tilde{\alpha}_{n+1}\right)\right)=2 \cdot \phi\left(\phi^{-1}\left(\tilde{\alpha}_{n+1}\right)\right)=2 \cdot \tilde{\alpha}_{n+1}=\tilde{\alpha}_{n}
$$


and

$$
\begin{aligned}
2^{m} \alpha_{n} & =2^{m} \cdot 2 \cdot \phi^{-1}\left(\tilde{\alpha}_{n+1}\right)=2 \cdot 2^{m} \cdot \phi^{-1}\left(\tilde{\alpha}_{n+1}\right) \\
& =2 \cdot \phi^{-1}\left(2^{m} \tilde{\alpha}_{n+1}\right)=2 \cdot \phi^{-1}\left(\tilde{\alpha}_{n-m+1}\right)=\alpha_{n-m} .
\end{aligned}
$$

Hence $\left\{\alpha_{n}\right\}$ is a divisible tower over $\alpha$ and $\alpha$ is a divisible element of $\operatorname{Br}(E)$.

If $\phi(\alpha)=0, \alpha$ lies in the kernel of $\phi$ and so is split by base change to $E l=l(u)$. Hence $\alpha$ is represented by the class of a central simple algebra with $E(\sqrt{d}) / E$ as a maximal subfield. Suppose that $\alpha=\epsilon_{1}$ for a quaternion algebra $\epsilon_{1}=(d, e)_{E}$ with $e \in E^{\times}$. Now since $k^{c y c}$ and $E$ are linearly disjoint over $k, k^{c y c} E$ is a $\mathbb{Z}_{2^{-}}$ extension of $E$ containing $E l$. Let $k_{n}^{c y c} E$ be the $\mathbb{Z} / 2^{n} \mathbb{Z}$ layer of $k^{c y c} E / E$, and choose generators $\sigma_{n} \in G_{k_{n}^{c y c} E / E}$ satisfying $\left.\sigma_{n}\right|_{k_{n-1}^{c y c} E}=\sigma_{n-1}$. Then the cyclic algebras $\epsilon_{n}=\left(k_{n}^{c y c} E / E,\left\langle\sigma_{n}\right\rangle, e\right)$ are each of order $2^{n}$ in $\operatorname{Br}(E)$ and, moreover, $2^{m} \epsilon_{n}=\epsilon_{n-m}$. Hence $\alpha$ is divisible in $\operatorname{Br}(E)$.

We have shown that $U_{2}(\omega 2, \operatorname{Br}(E))=0$. By [FSS1] and [FSS2], we have that $U_{2}(\omega 2, \operatorname{Br}(k(t)))=0$ and all other Ulm invariants of $\operatorname{Br}(E)$ and $\operatorname{Br}(k(t))$ are identical. Therefore $\operatorname{Br}(E) \cong \operatorname{Br}(k(t))$.

\section{ACKNOWLEDGMENTS}

The second author thanks the Department of Mathematics at the TechnionIsrael Institute of Technology for its hospitality during 1998-1999.

\section{REFERENCES}

[AB] M. Auslander and A. Brumer. Brauer groups of discrete valuation rings. Nederl. Akad. Wetensch. Proc. Ser. A 30 (1968), 286-296. MR 37:4051

[Br] A. Brumer. On the units of algebraic number fields. Mathematika 14 (1967), 121-124. MR 36:3746

[CR] C. Curtis and I. Reiner. Representation theory of finite groups and associative algebras. Pure and Applied Mathematics 11. New York: Wiley-Interscience, 1962. MR 26:2519

[Fa] D. K. Faddeev. Simple algebras over a field of algebraic functions of one variable. Trudy Mat. Inst. Steklov 38 (1951), 321-344; Amer. Math. Soc. Transl. II 3 (1956), 15-38. MR $17: 1046 \mathrm{e}$

[FS] B. Fein and M. Schacher. Brauer groups and character groups of function fields. J. Algebra 61 (1979), 249-255. MR 81c:12036

[FSS1] B. Fein, M. Schacher, and J. Sonn. Brauer groups of rational function fields. Bull. Amer. Math. Soc. (N.S.) 1 (1979), no. 5, 766-768. MR 80h:12019

[FSS2] B. Fein, M. Schacher, and J. Sonn. Brauer groups of fields of genus zero. J. Algebra 114 (1988), no. 2, 479-483. MR 89f:12008

[Ja] G. Janusz. Automorphism groups of simple algebras and group algebras. Representation theory of algebras (Proc. Conf., Temple Univ., Philadelphia, Pa., 1976). Lecture Notes in Pure Appl. Math. 37. New York: Dekker, 1978, pp. 381-388. MR 57:12610

[La] T. Y. Lam. The algebraic theory of quadratic forms, revised 2nd printing. Mathematics Lecture Note Series. Reading, Mass.: Benjamin/Cummings Publishing Co., Inc., 1980.

[NSW] J. Neukirch, A. Schmidt, and K. Wingberg. Cohomology of number fields. Grundlehren der mathematischen Wissenschaften 323. Berlin: Springer-Verlag, 2000. MR 2000j:11168

Department of Mathematics, Technion-Israel Institute of Technology, Haifa 32000 ISRAEL

E-mail address: sonn@math.technion.ac.il

Department of Mathematics, Davidson College, Box 7046, Davidson, North Carolina 28035-7046

E-mail address: joswallow@davidson.edu 